\title{
Positron Annihilation Studies of the Near-Surface Regions of Niobium before and after Wear Treatment
}

\author{
J. Dryzek ${ }^{1}$ (1) P. Horodek ${ }^{1,2}$
}

Received: 3 April 2017/Accepted: 31 July 2017/Published online: 16 August 2017

(c) The Author(s) 2017. This article is an open access publication

\begin{abstract}
The positron annihilation studies of well-annealed niobium samples after dry sliding or sandblasting are reported. These treatments modify surface and subsurface regions introducing crystalline defects, which are extended beneath the surface of the sample at a depth of about $140 \mu \mathrm{m}$ for the highest applied load of $50 \mathrm{~N}$. These defects can be identified as dislocations with jogs or vacancies near dislocations. The obtained depth profile of mean positron lifetime reflects the defect depth distribution and exhibits the characteristic plateau near the worn surface, which disappears when lowering of the applied load. A similar depth profile was obtained also for niobium samples after sandblasting. Slow positron beam studies revealed that at the surface of the samples after dry sliding or other treatments some defects that localize positrons are present. Additionally, a large value of positron diffusion length of about 266(33) nm was found for well annealed niobium.
\end{abstract}

Keywords Niobium · Dry sliding · Defect depth profiles · Positron annihilation techniques

\section{Introduction}

Treatment of surface of metals and alloys, i.e., machining, polishing, ion implantation, sandblasting, sliding, etc., induces elastic and plastic deformation, which create

\footnotetext{
J. Dryzek

jerzy.dryzek@ifj.edu.pl

1 Institute of Nuclear Physics Polish Academy of Sciences, 31342 Krakow, Poland

2 Joint Institute for Nuclear Research, Joliot-Curie 6, Dubna, Moscow Region, Russia 141980
}

crystalline defects in the region adjoining the surface $[1,2]$. This affects physical properties, for example, by causing an increase of hardness in this region. However, one can expect influence on electrical properties of conductors as well. It is well known that due to the skin effect, alternating electric current (AC) flows within a conductor near the surface. With the increasing frequency the current becomes distributed closer the surface, for instance in copper $1 \mathrm{MHz}$ AC current flows only at the depth of about $70 \mu \mathrm{m}$ from the surface. This depth coincidences with the depth of subsurface generated during dry sliding or sandblasting. In copper it is about $140-800 \mu \mathrm{m}$ as it was shown in our recent studies [3]. Thus, due to the skin effect conducting electrons are pushed to flow near the surface of a wire or a bar, but in this region they scatter at additional crystalline defects which may be created during manufacturing. This can be even more significant for RF cavities because the presence of surface and near surface defects causes increase of the surface resistance which affect the quality factor of a cavity, i.e., $Q_{0}$. This factor is defined as the ratio between the cavity stored energy and the power dissipated in the cavity walls in one RF radian, or it is proportional to the reciprocal of the surface resistivity of the wall material [4]. It was found that annealing at $110-120{ }^{\circ} \mathrm{C}$ is a necessary final stage in niobium superconducting RF cavity preparation to reach high gradients of electrical field [5] and improve of the $Q_{0}$ factor. It is called "baking effect" and some authors try to connect it with the presence of vacancies, dissociation of vacancy-hydrogen or vacancyoxygen complexes near the surface [6,7]. There have been several attempts to identify defects based on poor microwave performance at low temperatures in the superconductive state $[8,9]$. These authors indicate candidates for these defects such as pits, oxides, hydrides, impurity inclusions, grain boundaries, etc. The defects arise from 
cavity fabrication steps such as forming, machining, electron beam welding, electropolishing, and buffered chemical polishing.

Niobium is used for many special applications; it is an effective microalloying element for steel applied to increase the strength, formability, and weldability. The niobium-containing alloys and superalloys are important for its use in jet and rocket engines. Pure niobium as a superconducting material is used for construction of RF cavities for instance in the linear accelerators and free electron lasers.

There is also another aspect of studying niobium important for tribology. Our recent studies of subsurface zones created during dry sliding in $\mathrm{Ti}$ [10] and $\mathrm{Zr}$ [11] showed that they exhibited quite different defect depth distribution than in metals that have tribological application such as Fe [12], $\mathrm{Cu}$ [13], stainless steel [14]. We believe that studies of subsurface zone in metals, which are not used in tribojunctions can expand our knowledge about defects generation during sliding. Thus, studies on the subsurface region in pure niobium can be useful in this matter.

The aim of the paper is to recognize the types of defect and their depth distribution in pure niobium exposed to dry sliding and sandblasting processes. In our studies the conventional positron lifetime spectroscopy and slow positron beam will be used. This allows us to look at depths of 1 to hundreds of $\mu \mathrm{m}$.

\section{Experimental Details}

\subsection{Sample Preparation}

The initial preparation of our samples was similar to that in our former studies for other metals. The niobium samples of purity $99.9 \%$, purchased from Goodfellow, had a disc shape of $10 \mathrm{~mm}$ in diameter with $2 \mathrm{~mm}$ thickness. All samples before treatment were annealed in the flow of $\mathrm{N}_{2}$ gas at a temperature of $1000{ }^{\circ} \mathrm{C}$ for $1 \mathrm{~h}$ and then slowly cooled to room temperature. This value is much above the homologous temperature of recrystallization, which in pure metals is close to $0.3 \times T_{\mathrm{m}}$, where $T_{\mathrm{m}}$ is the melting point equal to $2750 \mathrm{~K}$ for niobium. (The recrystallization temperature is in the range of $899-1204{ }^{\circ} \mathrm{C}$, [15]). Additionally, all samples were chemically polished by a standard chemistry $(1: 1$ in volume mixture of $\mathrm{HF}: \mathrm{HNO}_{3}$ acids). About $50 \mu \mathrm{m}$ thick layer was etched. After this procedure only residual defects are present in the samples. In fact, in the positron lifetime spectrum measured for virgin samples, only one lifetime component equal to $129(1)$ ps was resolved. This value coincidences with the value reported in the literature, i.e., 128.3(3) ps for positron annihilation in bulk [16, 17].

\subsection{Sample Treatments}

After initial preparation described above, a sample was located in a tribotester, and the base surface of the sample slid against the rotating disc with an applied load. This disc of diameter $50 \mathrm{~mm}$ was made from the martensitic steel (steel SW18 hardness about $670 \mathrm{HV} 0.1$ ). The speed of the disc relative to the surface of the sample was equal to $5 \mathrm{~cm} / \mathrm{s}$. The treatment was performed in air during $1 \mathrm{~min}$, no oxidation was observed. The average value of the friction coefficient determined during the tribotest equals to $0.21 \pm 0.02$ and the specific wear rate, defined as the worn volume per unit sliding distance per unit of the load equals to $(9.68 \pm 0.07) \times 10^{-14} \mathrm{~m}^{3} \mathrm{~N}^{-1} \mathrm{~m}^{-1}$.

Another samples were sandblasted using Renfert Vario Basic Jet blaster. The abrasive material Edelkorund containing $99.8 \%$ alumina particles $\left(\mathrm{Al}_{2} \mathrm{O}_{3}\right)$ with size of $250 \mu \mathrm{m}$ in diameter was applied. The surfaces were blasted for $60 \mathrm{~s}$ under pressure of 4 and 6 bar with a distance of $10 \mathrm{~mm}$ between the sample and perpendicularly directed nozzle.

One more set of annealed samples was deformed by compression at room temperature to reduce their initial thicknesses. For compression, a uniaxial hydraulic press was used, and after $15 \mathrm{~s}$ the pressure was released.

\subsection{Positron Measurements}

Positron lifetime (LT) measurements were performed using fast-fast spectrometer based on the $\mathrm{BaF}_{2}$ scintillators. The timing resolution equaled 250 ps. The isotope ${ }^{22} \mathrm{Na}$ with activity of $32 \mu \mathrm{Ci}$ enveloped into two $7 \mu \mathrm{m}$ thick kapton foils was placed between two identical samples. The analysis of obtained spectra of $10^{6}$ counts was performed with LT code [18]. The diameter of the positron source spot is about $3 \mathrm{~mm}$, and it is smaller than the diameter of the samples.

The positron lifetime spectrum is described by the sum of exponential decay functions, characterized by the positron lifetime components, $\tau_{i}$ and their intensities $I_{i}$, where $i=1,2, \ldots N$ is the number of lifetime components. Usually, the number of components ranges from 1 to 4 depending on a sample. Each component is linked with the positron state at the moment of annihilation, i.e., the defect where a positron is localized and/or bulk region if it exists. For many applications the mean positron lifetime is used and it is defined as the sum: $\bar{\tau}=\tau_{1} I_{1}+\tau_{2} I_{2}+\cdots$. It is a robust parameter which does not depend on the number of components resolved from the positron lifetime spectrum; however, still it is sensitive to all parameters corresponding to the annihilation states and properties of the sample studied. 


\subsection{Positron Beam Measurements}

The reference sample, annealed and chemically polished, as well as surface-treated samples, were examined by Doppler spectroscopy (DB) using the variable energy positron beam (VEP) at JINR in Dubna. The monoenergetic positron flux with intensity of $10^{6} \mathrm{e}^{+} / \mathrm{s}$ was applied, its spot had a diameter of $5 \mathrm{~mm}$. Energy range of implanted positron was between 0.1 and $36 \mathrm{keV}$. The annihilation gamma radiation was recorded by a HPGe detector with energy resolution of $1.20 \mathrm{keV}$ interpolated for $511 \mathrm{keV}$. Each obtained spectrum was analyzed to calculate annihilation line shape parameter called $\mathrm{S}$ parameter, which is defined as the ratio of the area, of width of about $1.38 \mathrm{keV}$, below the central part of the annihilation line to the total area in the range of this line. The energy interval taken for calculation is always constant within the whole measurement session. It evaluates the fraction of positron-electron pairs with low momentum occurring mostly at open volume defects in the crystalline structure. The background under the annihilation was subtracted.

\section{Results and Discussion}

\subsection{Positron Lifetime Depth Profiles}

Theoretical calculations and experiments point out that the positron bulk lifetime in pure niobium is equal to 120 or 128.3(3) ps, respectively [19, 20]. For the simplest open volume defect, i.e., monovacancy in a perfect crystalline lattice the lifetime increases to 210 ps and for the vacancy clusters this value increase up to about $400 \mathrm{ps}$ [16]. In metals, plastic deformation introduces defects, mainly a tangle of dislocations and interfaces, i.e., grain boundaries, which affect mechanical and other physical properties. However, movement of dislocations and their interactions lead to generation of a great amount of point defects, such as vacancies and interstitial atoms [21]. The latter are mobile at low temperature and can recombine with vacancies, but some vacancies can survive. With the temperature increase vacancies can form clusters. Additionally, vacancies embedded in the local stress field can modify their size [22]. Nevertheless, open volume defects trap positrons and annihilation characteristics such as positron lifetime and/or S-parameter reflect their size and concentration, and this is the outline of our studies.

For deformed metals it is difficult to separate the certain type of defect using the positron experiments. For high deformation, all positrons after thermalization are trapped, and a certain value of positron lifetime is the result of the weighted sum over all trapped states. For a lower degree of deformation, i.e., lower strain, the situation is not simpler, because there is also annihilation in the bulk, thus nondeformed regions are possible. The experiments point out that with the increase of degree of deformation the mean positron lifetime increases, and this value saturates at high deformation. That is, for instance, for pure Fe where a lifetime component of about $150 \mathrm{ps}$ is observed for a high degree of deformation above $10 \%$ measured as thickness reduction in compression [23]. In the case of niobium it is similar. In Fig. 1 we depicted the dependency of the positron lifetime on the thickness reduction after compression. In all lifetime spectra a single lifetime component was resolved, and its value is depicted in Fig. 1. We have to point out that above $4 \%$ thickness reduction the saturation of the positron lifetime takes place at a value of about $180 \mathrm{ps}$, and this corresponds to the values obtained by other authors [16]. This value, lower than for monovacancy, indicates positron trapping at dislocations with jogs or monovacancies located near dislocations, squeezed in their elastic field (see Ref. [22] in analogy to the case of $b c c$ metal, e.g., Fe). Their density must be high even at a small degree of deformation because saturation of positron trapping is clearly observed. This can be explained if we compare the values of the yield strength, which is about $240 \mathrm{MPa}$ for niobium [24]. However, after annealing at $80{ }^{\circ} \mathrm{C}$ the yield strength decreases to $39.3 \mathrm{MPa}$ [25]. Thus, annealed niobium is more easily plastically deformed than $\mathrm{Zr}$ for which the yield strength is about $230 \mathrm{MPa}$ [24]. For Ti the yield strength ranges from 100 to $225 \mathrm{MPa}$ [24]. The

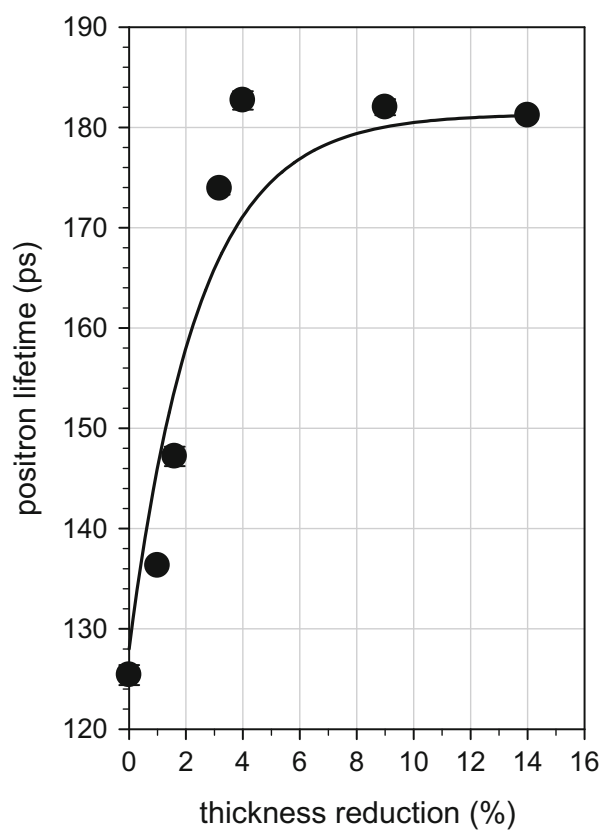

Fig. 1 The measured values of the positron lifetime versus thickness reduction of compressed well annealed niobium. The solid line represents the best fit of the following equation: $\tau=\tau_{\text {sat }}+\left(\tau_{\text {bulk }}-\tau_{\text {sat }}\right)$ $\exp (-c \varepsilon)$ where $\tau_{\text {sat }}=181(7) \mathrm{ps}, \tau_{\text {bulk }}=128(8) \mathrm{ps}, c=0.41(18)$ and $\varepsilon$ is the thickness reduction expressed in \% 
obtained dependency one can try to describe using an exponential function, as seen in Fig. 1 represented by the solid line.

During dry sliding of two bodies, plastic deformation occurs at asperities or in their vicinity; however, experiments show that this also occurs in their interior. For scanning of this region we performed measurements of the positron lifetime in a sequenced etching procedure as follows. The niobium samples after dry sliding were sequentially etched to remove the layer of about $15 \mu \mathrm{m}$ thick from the worn surface and the measurement of positron lifetime spectrum was performed after removing each layer. It was found that chemical etching does not introduce any defects that could disturb the initial defect depth distribution generated during sliding. This is because the solution interacts only with atoms at the surface, not in the interior, at a depth where positrons are implanted. In our studies the samples were etched in the solution described in Sect. 2.1. The thickness of the sample was measured using a digital micro-screw with accuracy $\pm 1 \mu \mathrm{m}$. One can add that in niobium the average implantation depth of a positron emitted from ${ }^{22} \mathrm{Na}$ is about $26 \mu \mathrm{m}$, i.e., about $63 \%$ positrons annihilate in the layer of this thickness. In Fig. 2 we depicted the measured values of the mean positron lifetime for three values of the load applied during sliding, i.e., 5, 25, and $50 \mathrm{~N}$. However, the depicted results must be carefully described.

For a load of $50 \mathrm{~N}$ the value the single lifetime component was resolved for the depth range up to $100 \mu \mathrm{m}$; its value decreases from 175.8(6) ps to 160.1(3). This indicates that only defects associated with dislocations are present at this depth. For vacancy clusters the positron lifetime would be much above 210 ps [16]. At deeper depths, i.e., in the range of 100 to $170 \mu \mathrm{m}$, two lifetime

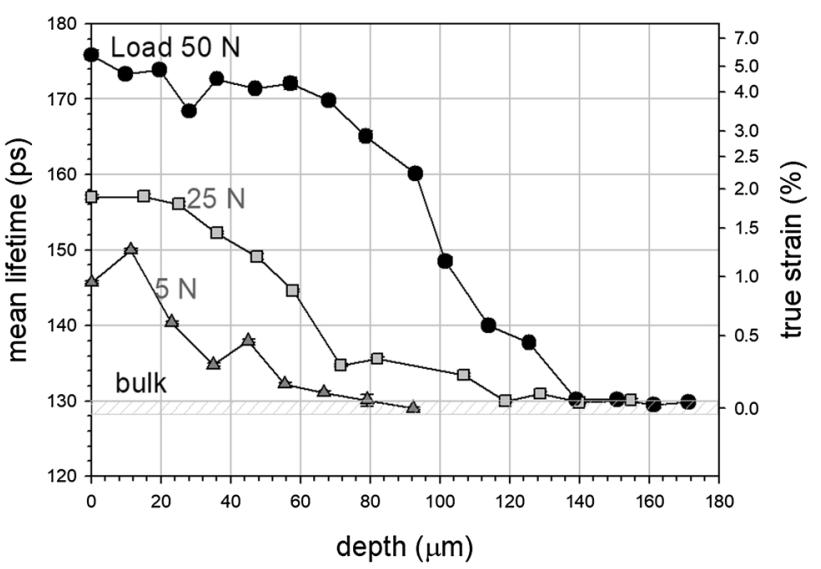

Fig. 2 Mean positron lifetime (left axis) versus depth from the worn surface of the niobium samples exposed to the dry sliding. Three values of the load of 5,15 , and $50 \mathrm{~N}$ were used. On the right axis the true strain calculated from the dependency in Fig. 1 is tagged (see text). The dashed region represent the bulk positron lifetime components were resolved. The first value was equal to about $60 \mathrm{ps}$ and the second one to about $155 \mathrm{ps}$. This indicates that the defect-free(bulk) regions are present also according the so-called positron trapping model [26]. However, dislocations are present, as well as indicated by the presence of the second lifetime component in the positron lifetime spectrum. The total range of the deformed region induced by sliding for this load is about $140 \mu \mathrm{m}$. Beyond this range only single lifetime component close to the bulk value is observed in the positron lifetime spectra. The profile touches the hatched region in Fig. 2. No vacancy clusters, frequently observed in other deformed metals are present.

It is worth noticing that in the layer with a thickness of about $60 \mu \mathrm{m}$ adjoining the worn surface the mean positron lifetime almost does not alter. This plateau was observed in similar studies for $\mathrm{Ti}$; however, in that case vacancy clusters were detected. This can be explained as follows. Ti has a hexagonal crystalline structure with fewer sliding systems in comparison to the $b c c$ structure of niobium. Additionally, the melting point for $\mathrm{Ti}$ is much lower than for niobium. Thus, in the latter case one expects dislocations rather than vacancy clusters. However, it can be mentioned that a certain number of vacancy clusters in niobium exposed to high pressure torsion was observed as well [16]. The range of the plateau is lower, i.e., up to $20 \mu \mathrm{m}$ for the sample exposed to the sliding with a lower load of $25 \mathrm{~N}$, and it disappears for the lowest load of $5 \mathrm{~N}$; see Fig. 2. The presence of the plateau can be explained by the dependency depicted in Fig. 1. If the deformation of the layer adjoining the worn surface is higher than about $4 \%$ the positron trapping saturates.

Linking data from Figs. 1 and 2 we can evaluate the true strain profile below the worn surface. The relation linking the mean positron lifetime and the thickness reduction described by the solid line serves as scaling of the right $y$ axis in Fig. 2 showing true strain values; for details see Ref. [11]. It can be noticed that with the depth increase the strain decreases; however, the obtained dependency cannot be described using an exponential decay function like for $\mathrm{Zr}$ [11]. As found in the previous studies, the applied load has a great impact on the total range of the deformed zone. It is about $140 \mu \mathrm{m}$ for a load of $50 \mathrm{~N}, 120 \mu \mathrm{m}$ for $25 \mathrm{~N}$, and $70 \mu \mathrm{m}$ for the lowest load of $5 \mathrm{~N}$.

In comparison to $\mathrm{Zr}$ and $\mathrm{Ti}$, the saturation of the positron lifetime for niobium is achieved at lower deformation degree. However, the type of depth profile of the positron lifetime in the subsurface zone is similar to both of those metals.

In Fig. 3 we depicted the depth profiles of the mean positron lifetime for well annealed samples exposed to sandblasting with alumina particles at pressures of 4 and 6 bar. The striking feature of the results is that the obtained 


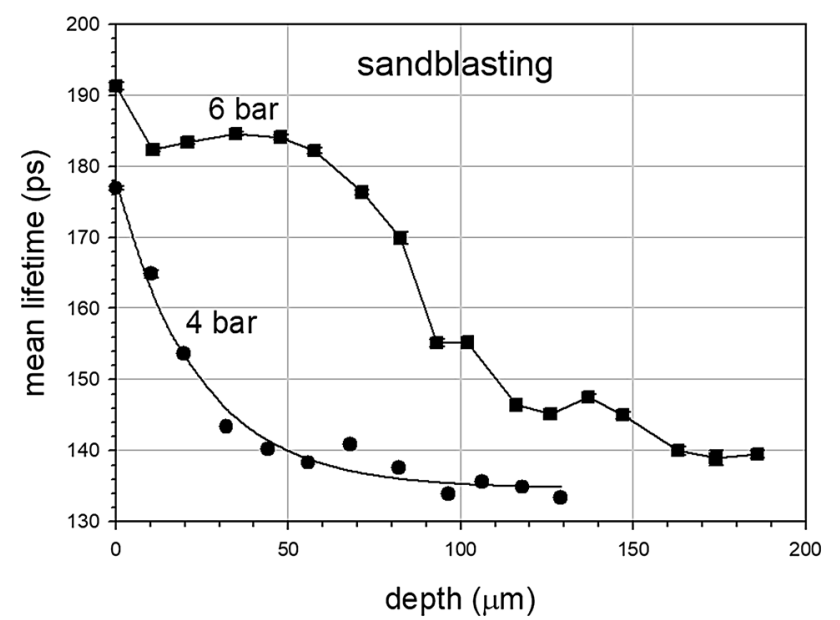

Fig. 3 Mean positron lifetime versus depth from the surface of the niobium samples exposed to sandblasting with the alumina particles with pressures of 4 and 6 bar

profiles almost reproduce the results from Fig. 2 for the lowest and highest load. These can be recognized as the characteristic plateau and exponential decay dependency as well. This shows us that mechanisms of damage in the region below the worn surface for dry sliding and sandblasting are very similar, despite different forces acting on the surface, i.e., normal impact only in the latter case. We argue that impacts during dry sliding play a significant role in generation of deformation in the subsurface region.

\subsection{Positron Beam Results}

Positron lifetime spectrum is sensitive only to open volume defects, which additionally must localize positrons. However, it is not sensitive to defects such as interstitial atoms or fault tetrahedrons, which can be created as well. The VEP technique can supplement this information because it is sensitive to the positron diffusion process. Generally, defects can scatter randomly walking positrons then they affect the positron diffusion coefficient and finally the positron diffusion length determine in VEP experiment, see "Appendix". Additionally this technique is sensitive to the surface state or surface defects, which act as traps for positrons returning from the interior. Thus, this technique can deliver the unique information not available using other positron techniques.

The S-parameter values as a function of positron incident energy in VEP measurements for selected niobium samples are depicted in Fig. 4. The closed circles represent the results for the reference well annealed sample, Fig. 4a. With the increasing impact energy the S-parameter value decreases and it is a characteristic feature of many metals. This dependency reflects the fact that positrons after implantation and thermalization walk randomly, and during this process they can also return to the entrance surface. Positrons can be mean positron implantation depth $(\mathrm{nm})$

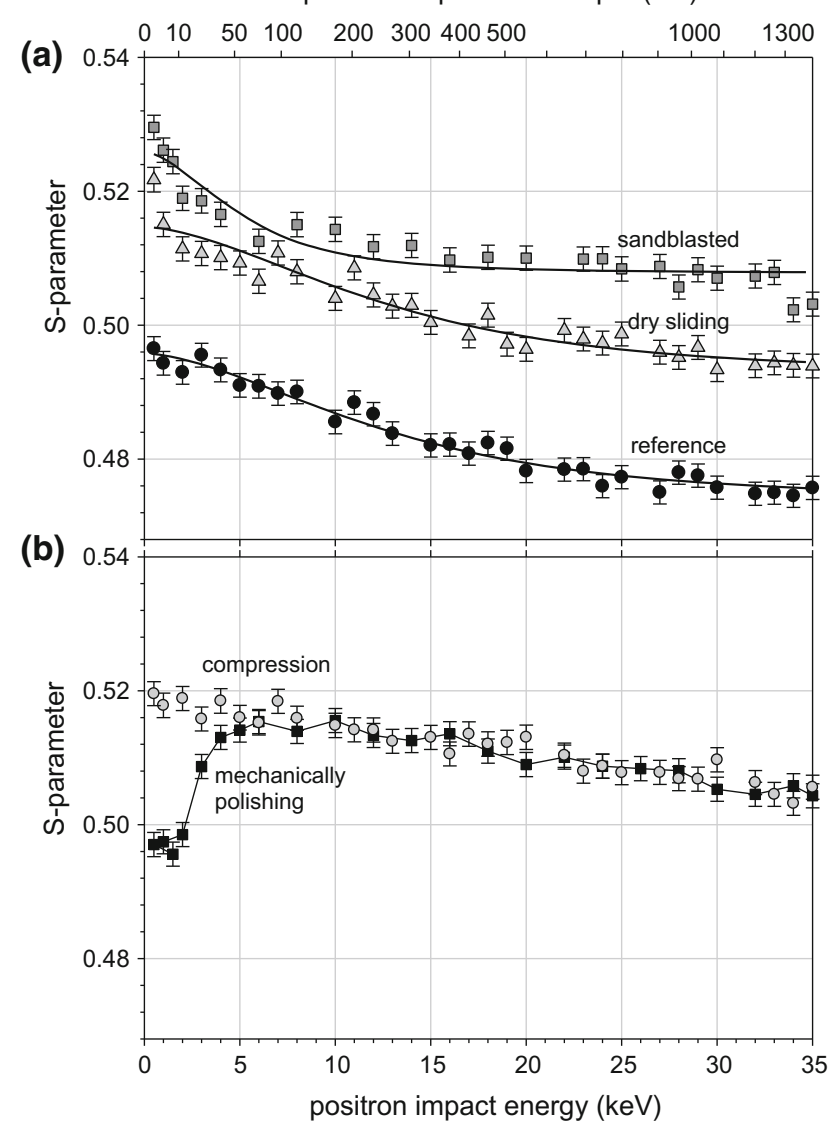

Fig. 4 The S-parameter versus the positron impact energy in VEP measurements for the reference well annealed sample (closed circles), sample exposed to sandblasting with alumina particles at a pressure of 6 bar (grey squares) and exposed to dry sliding with an applied load of $50 \mathrm{~N}$ (grey triangles). On the top axis the mean positron implantation depth calculated using relation Eq. A2 is tagged. The solid lines in a represent the best fit of Eq. A3 to the experimental points. In $\mathbf{b}$ the S-parameter dependency for compressed niobium samples with pressure of $22 \mathrm{MPa}$ (grey circles) and with a subsequent polishing surface (see text) (black squares)

trapped at the surface where the electron density is lower than in the bulk. Additionally, they can be trapped at surface defects or positronium, i.e., bound state between positron and electron can be created. All of this causes that the value of the S-parameter at the surface can be higher than in the bulk. The dependency of the S-parameter on the positron impact energy can be described by Eq. (A3), see "Appendix". From the Monte Carlo simulations using GEANT 4 codes we obtained the parameters, which describe positron implantation profile, Eq. (A1), and hence the values of the mean positron implantation range are calculated and noted on the top-axis, Fig. 4. The solid lines in Fig. 4a represent the best fit of Eq. (A3) to the experimental points. Only three adjustable parameters were used in this procedure. Their values, in the case of the reference sample, are equal to $S_{\text {int }}=S_{\text {bulk }}=0.474(1), S_{\text {surf }}=0.496(1)$, and the positron 
diffusion length: $L_{+}=266(33) \mathrm{nm}$. This is a larger value in comparison to other metals, for instance in $\mathrm{Cu}$ it is equal to 121(7) $\mathrm{nm}$ [13] and in stainless steel 73.7(1.7) nm [27]. Nevertheless, this value coincides with data from the literature, Čížek et al. reported even higher value, i.e., about 319(8) nm [17].

For the sample exposed to dry sliding with the load of $50 \mathrm{~N}$ the determined values are equal to: $S_{\text {int }}=0.493(1)$, $S_{\text {surf }}=0.515(1)$, and the positron diffusion length: $L_{+}=266(58) \mathrm{nm}$. Despite the defects present in the layer adjoining the worn surface, noted by the increase of the $S_{\text {int }}$ value, no reduction of the positron diffusion length is observed, see Eqs. (A3) and (A4). However, introduction of more defects, as in the case of sandblasted sample induces reduction of diffusion length, $L_{+}=110(36) \mathrm{nm}$. For this sample, the other adjustable parameters are equal to: $S_{\text {int }}=0.507(1), S_{\text {surf }}=0.526(1)$.

One should emphasize the significant increase of the value of the $S_{\text {surf }}$ parameter for the samples after surface treatments in comparison to the reference sample. This is the characteristic feature which was not observed for instance, for $\mathrm{Cu}$ [13] or Fe [28], where the $S_{\text {surf }}$ values do not change significantly after surface treatment. This suggests that a new type of defect or defects, presumably of larger size, are created at the surface of niobium samples. In other words, the surface of niobium is sensitive to the treatment. This can be seen in Fig. 4b, where the results for the compressed sample (open circles) and the sample after compression with the surface mechanically polished using fine grained abrasive papers grade of $\mathrm{P} 1000$ and $\mathrm{P} 1200$ to get a shine before VEP measurements (closed squares) are presented. It can be seen that for the latter $S_{\text {surf }}=0.497(2)$. Then its value drops to the value obtained for the reference sample, see Fig. 4a. It is worth noticing that VEP results obtained for niobium are significantly different than those obtained in similar conditions for $\mathrm{Cu}$ [13], Fe [28], or stainless steel [14, 27]. Nevertheless, they are in qualitative agreement with the results obtained by $\mathrm{Gu}$ et al. [29]. Those authors investigated niobium samples exposed to dry sliding using VEP as well. For the samples exposed to much higher loads, i.e., 490 and $784 \mathrm{~N}$ the decrease of the S-parameter below $6 \mathrm{keV}$ takes place. However, these authors used much lower positron impact energy less than $18 \mathrm{keV}$, making analysis of their data impossible using for instance Eq. (A3).

\section{Remarks}

Dry sliding causes plastic deformation and large strain below the worn surface, which is distributed to a certain depth. The deformation is so high that the positron trapping saturates and the characteristic plateau close to the surface is observed. The presence of the plateau in the positron lifetime dependency in the layer adjoining the worn surface seems to be more general than the exponential decay observed previously, for instance for $\mathrm{Cu}$ [13]. Nevertheless, the plateau can disappear and the exponential decay occurs when a lower load is applied and less strain is generated. All types of the mean positron lifetime profiles one can find in Fig. 2. We argue that direct at the worn surface positrons are trapped at the open-volume defects created during sliding. Presumably in $\mathrm{Cu}$ and $\mathrm{Fe}$ defects at the surface are annealed during sliding and in these cases the S-parameter at the surface before and after sliding does not significantly differ. Niobium in our experiments exhibited much lower wear rate against the martensitic steel than Ti, i.e., $(4.23 \pm 0.05) \times$ $10^{-13} \mathrm{~m}^{3} \mathrm{~N}^{-1} \mathrm{~m}^{-1}$ [10] or Fe, i.e., $(5.58 \pm 0.11) \times 10^{-13}$ $\mathrm{m}^{3} \mathrm{~N}^{-1} \mathrm{~m}^{-1}$ [12] measured in the identical conditions. Thus, niobium after the surface treatments exhibits similar defect depth distribution as other metals studied, nevertheless high surface sensitivity to the positron annihilation is a new feature of this metal. This was also observed by Romanenko and Grassellino who measured the microwave surface resistivity of superconducting niobium at RF field [30]. This resistivity strongly increases in chemically treated niobium, presumably due to different roughness. We would argue that some defects, which strongly localize positrons can be created at the surface and are responsible for the microwave surface resistivity.

\section{Conclusions}

Positron annihilation studies of subsurface zone in pure niobium samples exposed to dry sliding revealed the defect depth profile extended in the range from 70 to $140 \mu \mathrm{m}$ depending on the applied load in the range from 5 to $50 \mathrm{~N}$. The characteristic feature of the measured positron mean lifetime depth profile is the plateau located near the worn surface. This plateau disappears for the lowest load applied. The subsurface zone contains dislocations that jog, which localizes positrons. No vacancy clusters were detected. Similar defect depth profiles were found for the sandblasted niobium samples. Slow positron beam measurements pointed out that at the surface of niobium samples exposed to dry sliding, sandblasting, and compression, some defects localizing positrons are present. These defects enhance the value of the S-parameter at the surface significantly in comparison to the value for the reference sample.

Open Access This article is distributed under the terms of the Creative Commons Attribution 4.0 International License (http://crea tivecommons.org/licenses/by/4.0/), which permits unrestricted use, distribution, and reproduction in any medium, provided you give appropriate credit to the original author(s) and the source, provide a link to the Creative Commons license, and indicate if changes were made. 


\section{Appendix}

Positrons with energy $E$ injected into a sample are distributed at a certain depth from the entrance surface. Then they begin the random walk with the thermal energy until annihilation. According to the Monte Carlo simulations the positron implantation profile can be described using the Makhovian function as follows:

$p(x, E)=\frac{m x^{m-1}}{x_{0}^{m}} \exp \left[-\left(\frac{x}{x_{0}}\right)^{m}\right]$.

where

$x_{0}=\frac{A_{1 / 2}}{\rho(\ln 2)^{1 / m}} E^{n}$,

$\rho$ represents the density of the implanted sample. The value of the parameters, i.e., $n, m$ and $A_{1 / 2}$, can be found by adjusting the function $\mathrm{A} 1$ to the simulated implantation profile; see Ref. [31]. Using GEANT 4 codes we found that for pure $\mathrm{Nb}: n=1.662, A_{1 / 2}=2.61 \mu \mathrm{g} / \mathrm{cm}^{2} \mathrm{keV}^{n}$ and $m=1.733$, this value was obtained after averaging the values obtained for energy $E$ in the range from 1 to $40 \mathrm{keV}$. It is necessary because the $m$ parameter changes slightly with the change of impact energy. The mean positron implantation depth is as follows:

$\bar{x}=x_{0} \Gamma(1+1 / m)$,

where $\Gamma$ is the gamma function.

Random walk can be terminated in the interior below the surface, but also at the entrance surface. Let us denote that value of the S-parameter for positrons annihilating at the surface is $S_{\text {surf }}$, and for annihilation in the interior $S_{\text {int }}$. The value of the measured S-parameter must be averaged over all possible annihilation states, thus taking into account the diffusion equation and assumption that positrons are not reflected from the surface, one can describe this value as follows:

$S(E)=S_{\text {bulk }}+\left(S_{\text {surf }}-S_{\text {int }}\right) \int_{0}^{\infty} \mathrm{d} x p(x, E) \exp \left(-x / L_{+}\right)$,

where $L_{+}$, is the positron diffusion length. This value depends on the positron diffusion coefficient $D_{+}$and annihilation rate $\lambda$ in the interior as follows: $L_{+}=\sqrt{D_{+} / \lambda}$. If the interior contains only one type of open volume defect which can trap positrons with the rate $k_{0}$ the annihilation rate is equal to:

$\lambda=\lambda_{\text {bulk }}+k_{0}$,

and
$S_{\text {int }}=\frac{S_{\text {def }} k_{0}+S_{\text {bulk }} \lambda_{\text {bulk }}}{\lambda_{\text {bulk }}+k_{0}}$,

where $\lambda_{\text {bulk }}, S_{\text {bulk }}$, represent the values of the annihilation rate and the S-parameter for the bulk, free of defects region and $S_{\mathrm{def}}$, the value of the S-parameter for annihilation of positrons trapped at the defect. In this studies we neglected the ephithermal positrons emitted from the surface, which sometimes are included into analysis. Usually, they are not sensitive to the defects or sample structure. Also, backscattering of positrons diffused from the interior at the surface is neglected.

\section{References}

1. Zum Gahr, K.-H.: Microstructure and Wear of Materials. Elsevier, Amsterdam (1987)

2. Hutchings, I.M.: Tribology: Friction and Wear of Engineering Materials. CRC Press, Boca Raton (1992)

3. Horodek, P., Siemek, K., Dryzek, J., Wróbel, M.: Positron annihilation and complementary studies of copper sandblasted at differen. (submitted to Wear)

4. Ciovati, G., Halbritter, J.: Analysis of the medium field Q-slope in superconducting cavities made of bulk niobium. Phys. C 441, 57-61 (2006)

5. Visentin, B., et al.: Proceedings of the 6th EPAC, Stockholm vol. III. p. 1885 (1998)

6. Visentin, B., Barthe, M.F., Moineau, V., Desgardin, P.: Positron annihilation spectroscopy on Niobium samples. In: 14 th International Conference on RF Superconductivity-Berlin (Germany)-Sept. 21 st to 25 th,-TUPPO047 (2009)

7. Visentin, B., Gasser, Y., Charrier, J.P.: First results on fast baking. Phys. C 441, 66-69 (2006)

8. Nishizaki, T., Lee, S., Horita, Z., Sasaki, T., Kobayashi, N.: Superconducting properties in bulk nanostructured niobium prepared by high-pressure torsion. Phys. C 493, 132-135 (2013)

9. Tai, T., Ghamsari, B.G., Bieler, T., Steven, Anlage M.: Nanoscale nonlinear radio frequency properties of bulk $\mathrm{Nb}$ : origins of extrinsic nonlinear effects. Phys. Rev. B 92, 134513 (2015)

10. Dryzek, J., Wróbel, M.: Positron studies of subsurface zone in Titanium created in sliding wear. Tribol. Lett. 55, 413-419 (2014)

11. Dryzek, J., Siemek, K.: Formation of subsurface zone induced by sliding wear in Zirconium studied by positron lifetime spectroscopy. Tribol. Lett. 64, 15 (2016)

12. Dryzek, J.: Positron studies of subsurface zone in pure iron induced by sliding. Tribol. Lett. 42, 9-15 (2011)

13. Dryzek, J., Dryzek, E., Börner, F., Krause-Rehberg, R.: Positron annihilation studies of subsurface zones in copper created under lubrication conditions. Mater. Sci. Forum 363-365, 517 (2001)

14. Horodek, P., Siemek, K., Dryzek, J., Kobets, A.G., Wróbe, M.: Positron annihilation and complementary studies of stainless steel exposed to sandblasting at different angles. Tribol. Lett. 65, 30 (2017)

15. Gupta, C.K., Suri, A.K.: Extractive Metallury of Niobium. CRC Press. Inc, Boca Raton (1994)

16. Čížek, J., Melikhova, O., Barnovská, Z., Procházka, I., Islamgaliev, R.K.: Vacancy clusters in ultrafine grained metals prepared by severe plastic deformation. J. Phys: Conf. Ser. 443, 012008 (2013) 
17. Č́ížek, J., Procházka, I., Brauer, G., Anwand, W., Gemma, R., Nikitin, E., Kirchheim, R., Pundt, A.: Hydrogen interaction with vacancies in electron irradiated Niobium. Act. Phys. Polon. A 113, 1293 (2008)

18. Kansy, J.: Microcomputer program for analysis of positron annihilation lifetime spectra. Nucl. Instrum. Methods Phys. Res. A 374, 235-244 (1996)

19. Campillo Robles, J.M., Ogando, E., Plazaola, F.J.: Positron lifetime calculation for the elements of the periodic table. J. Phys.: Condens. Matter 19, 176222 (2007). doi:10.1088/0953-8984/19/ $17 / 176222$

20. Čížek, J., Procházka, I., Becvář, F., Kužel, R., Cieslar, M., Brauer, G., Anwand, W., Kirchheim, R., Pundt, A.: Hydrogeninduced defects in bulk niobium. Phys. Rev. B 69, 224106 (2004)

21. Hull, D.: Introduction to Dislocations. Pergamon Press, Oxford (1975)

22. Kamimura, Y., Tsutsumi, T., Kuramoto, E.: Calculations of positron lifetimes in a jog and vacancies on an edge-dislocation line in Fe. Phys. Rev. B 52, 879-885 (1995)

23. Somieski, B., Krause-Rehberg, R.: Application of the positron lifetime spectroscopy as a method of non-destructive testing. Mater. Sci. Forum 175-178, 989-992 (1995)

24. Goodfellow Cambridge Ltd. http://www.goodfellow.com
25. Myneni, G.R., Umezawa, H.: Variation of mechanical properties of high RRR and reactor grade niobium with heat treatments. In: Conference Lectured at the 10th International Conference on Ultra-High Purity Metallic-Base Materials (UMPH 2003)

26. Dryzek, J., Kansy, J.: Comparision of three programs: positronfit, resolution and LT for deconvolution of positron lifetime spectra. Nucl. Instrum. Methods Phys. Res. A 380, 576 (1996)

27. Dryzek, J., Horodek, P.: The solution of the positron diffusion trapping model tested for profiling of defects induced by proton implanted in stainless steel. Appl. Phys. 121A, 289-295 (2015)

28. Dryzek, J., Dryzek, E., Krause-Rehberg, R., Redman, F.: Subsurface zones in steel samples studied by means of positron annihilation. Tribol. Lett. 11, 121 (2001)

29. Gu, H.D., Wang, T.M., Wang, W.J., Leung, K.M., Chung, C.Y.: Slow positron annihilation studies of vacancy-type defects in the near-surface region of $\mathrm{Cu}$ and $\mathrm{Nb}$ before and after wear. Appl. Phys. A 68, 325-327 (1999)

30. Romanenko, A., Grassellino, A.: Dependence of the microwave surface resistance of superconducting niobium on the magnitude of the rf field. Appl. Phys. Lett. 102, 252603 (2013)

31. Dryzek, J., Horodek, P.: GEANT4 simulation of slow positron beam implantation profiles. Nucl. Instrum. Methods Phys. Res. B 266, 4000-4009 (2008) 